\title{
A Comparison of Artificial Neural Network and Decision Trees with Logistic Regression as Classification Models for Breast Cancer Survival
}

\author{
Venkateswara Rao Mudunuru \\ Department of Mathematics and Statistics, \\ University of South Florida, Tampa, FL, USA. \\ Corresponding author: vmudunur@mail.usf.edu \\ Leslaw A. Skrzypek \\ Department of Mathematics and Statistics, \\ University of South Florida, Tampa, FL, USA. \\ E-mail: skrzypek@usf.edu
}

(Received April 10, 2020; Accepted July 3, 2020)

\begin{abstract}
In the field of medicine, several recent studies have shown the value of Artificial Neural Networks, decision trees, logistic regression are playing a major role as the predictor, and classification methods. The research has been expanded to estimate the incidence of breast, lung, liver, ovarian, cervical, bladder and skin cancer. The main aim of this paper is to develop models of logistic regression, Artificial Neural Networks, and Decision trees using the same input and output variables and to compare their success in predicting breast cancer survival in woman. To find the best model for breast cancer survival, the sensitivity and specificity of all these models are measured and evaluated with their respective confidence intervals and the ROC values.
\end{abstract}

Keywords- Artificial neural networks, Logistic Regression, Breast cancer, Decision Trees, Cancer survival.

\section{Introduction}

In the field of clinical diagnostics, computer models are playing a prominent role in differentiating between a healthy and an ill patient. Such computer model accuracy is held accountable for encouraging correct decisions about the risk of disease based on the patient's characteristics. Numerous data models designed, assessed and improved include both statistical methods as well as non-statistical ones. Every methodology uses different assumptions and, depending on the data context, may or may not produce similar results. Three frequently used methods are regression, decision trees, and artificial neural networks. Regression methodology estimates the relation between the independent and dependent variables. Regression methods are also referred to as dependence analysis techniques (Agresti, 2010). Study of regression models is a crucial part of several research projects. Such models are widely applied in order to assess the survival of severely ill patients admitted to the intensive care unit (Gellar et al., 2014). Linear models and logistic regression models are two main categories of regression methods. The technique of logistic regression is very widely used in data analysis. It is considered a well-known model of classification that enables probabilistic decisions to be made and shows promising results on several issues. A logistic model is often considered a clinically interpretable model for providing best-fit similar results.

Four models are developed in this paper considering the same output and the set of input variables using decision trees, logistic regression and artificial neural networks. Performance of these models is assessed in breast cancer survival prediction. The four models are developed by considering 
International Journal of Mathematical, Engineering and Management Sciences

Vol. 5, No. 6, 1170-1190, 2020

https://doi.org/10.33889/IJMEMS.2020.5.6.089

cancer data available in SEER. Surveillance, Epidemiology and End Results (SEER) is the most widely used Medicare database for cancer datasets. This data has been pre-processed (cleansed) to eliminate or account for missing data and repetitions. The final data set considered in this research contained 47,167 records of malignant tumors (Mudunuru, 2016).

In this paper, the survival of a breast cancer woman is estimated using important available attributable variables in the database. Initially, we selected all the independent variables including size of the tumor, age of the patient, stage of the breast cancer, treatment administered, duration, tumor grade, marital status of the patient, and the count of primary tumors recorded. Logistic regression eliminated variables like size of the tumor, grade of the cancer, marital status of the patient to be statistically insignificant in prediction survival of women with breast cancer. We developed our four models by inputting only one of the remaining significant variables at a time. All these models provided us with an output vector with two variables for each case: either 1 (alive/ survived) or 0 (dead/ not survived). A number ranging between 0 and 1 is used to estimate the accuracy of predicted value.

\section{Logistic Regression}

One of the primary assumptions of linear logistic regression is that the independent covariates are in linear association with their corresponding natural logarithm of odds. The three main components that define logistic function are a systematic part, the related link function and the random experiment. The binary nature of the dependent variable that is eminently suited for modelling data is also another important characteristic of logistic function. As a result of this, the target of logistic regression therefore is slightly different since we estimate the probability that the response variable is equal to provided values of independent variables (McFadden, 1973).

Survival prediction of breast cancer is the output variable in our current research from the given patient's age, size of tumor, stage of cancer, treatment administered, and the duration.

\subsection{Survival Prediction using Logistic Modelling}

The two most general and widely used survival analyses techniques are Event history models and logistic regression. Survival time is considered as a continuous variable in the event history model whereas it is considered as discrete in logistic regression models. A dichotomous measure (survived or not) is thus the target. Logistic regression model as a classifier is applied in this section to predict breast cancer survival in women.

Age and size of the tumor are used in the first model (model-1). Age, size of the tumor and stage of the cancer are used in the second model (model-2). Along with the three variables selected in model-2, treatment is added to develop the third model (model-3). Age, size of the tumor, cancer stage, treatment and duration are all used in the fourth model (model-4). Our primary aim is to measure or estimate the women's breast cancer survival.

The overall accuracy of the models is attributed as a reliability measure of the given estimate. Specificity, sensitivity along with $95 \%$ confidence intervals and overall accuracy for the developed logistic models are given in the Table 1. 
International Journal of Mathematical, Engineering and Management Sciences

Vol. 5, No. 6, 1170-1190, 2020

https://doi.org/10.33889/IJMEMS.2020.5.6.089

Table 1. Sensitivity, specificity and overall results of Logistic regression models

\begin{tabular}{|l|c|c|c|c|c|}
\hline \multirow{2}{*}{$\begin{array}{l}\text { Logistic Regression } \\
\text { Models }\end{array}$} & Value & $\begin{array}{c}|c| \\
\text { Confidence } \\
\text { Interval }\end{array}$ & Value & $\begin{array}{c}\text { Sensitivity } \\
\text { Confidence } \\
\text { Interval }\end{array}$ & \multirow{2}{*}{ Accuracy } \\
\hline Logistic model 1 & $68.01 \%$ & $67.04-68.96$ & $69.45 \%$ & $68.99-69.91$ & $69.2 \%$ \\
\hline Logistic model 2 & $67.31 \%$ & $66.39-68.21$ & $70.47 \%$ & $70.00-70.93$ & $69.78 \%$ \\
\hline Logistic model 3 & $67.69 \%$ & $66.80-68.55$ & $71.26 \%$ & $70.78-71.72$ & $70.42 \%$ \\
\hline Logistic model 4 & $76.82 \%$ & $76.14-77.47$ & $81.54 \%$ & $81.11-81.97$ & $79.98 \%$ \\
\hline
\end{tabular}

The ROC area values along with specificity at $95 \%$ sensitivity for the developed logistic models are given in Table 2 .

Table 2. ROC Areas for Logistic models

\begin{tabular}{|l|c|c|}
\hline \multirow{2}{*}{$\begin{array}{l}\text { Logistic Regression } \\
\text { Models }\end{array}$} & \multirow{2}{*}{ ROC } & At 95\% Sensitivity \\
\cline { 3 - 3 } & & Specificity \\
\hline Logistic model 1 & $68.8 \%$ & $25 \%$ \\
\hline Logistic model 2 & $71.0 \%$ & $30 \%$ \\
\hline Logistic model 3 & $71.8 \%$ & $29 \%$ \\
\hline Logistic model 4 & $85.5 \%$ & $61 \%$ \\
\hline
\end{tabular}

The results showed that for model-3, the overall accuracy is $70.42 \%$ that increases to $80 \%$ for model-4. As expected, the tumor duration has large significance for predicting accurate survival during the study period. The ROC area of logistic regression model-1 is $68.8 \%$ and a survival sensitivity of $95 \%$ yielded a specificity of just $25 \%$, model- 2 on the other hand with a ROC area of $71 \%$ and survival sensitivity of $95 \%$ provided $30 \%$ specificity rate. The ROC area values for the remaining two models are $71.8 \%$ and $85.5 \%$ respectively, and the $95 \%$ survival sensitivity rate has a $29 \%$ and $61 \%$ overall specificity, respectively. Highly attractive is the performance of fourth logistic model offering $80 \%$ overall accuracy. The specificity and sensitivity for this model are $81.54 \%, 76.82 \%$ respectively. At a $95 \%$ sensitivity this model has $61 \%$ specificity. Figure 1 displays the ROC graphs for the logistic models. 
International Journal of Mathematical, Engineering and Management Sciences

Vol. 5, No. 6, 1170-1190, 2020

https://doi.org/10.33889/IJMEMS.2020.5.6.089

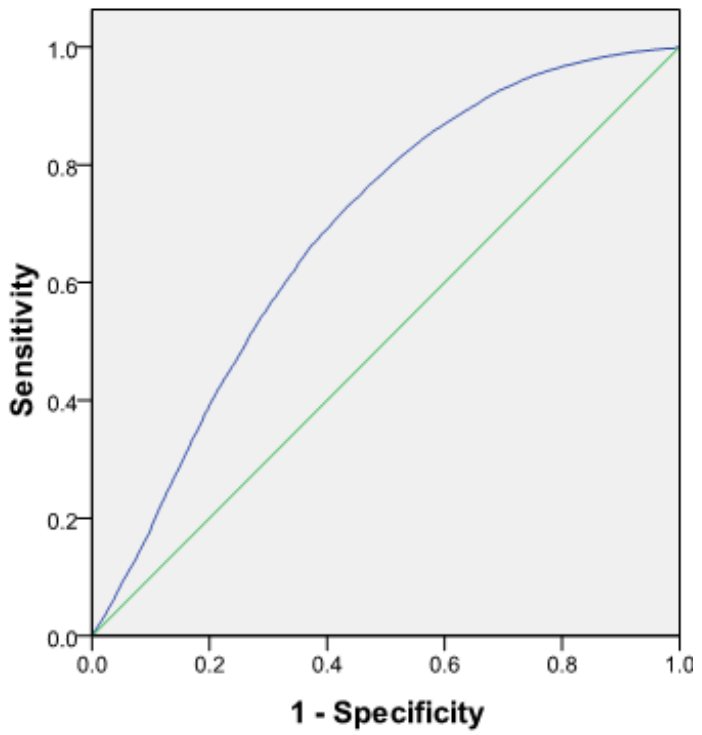

Logistic Model 1

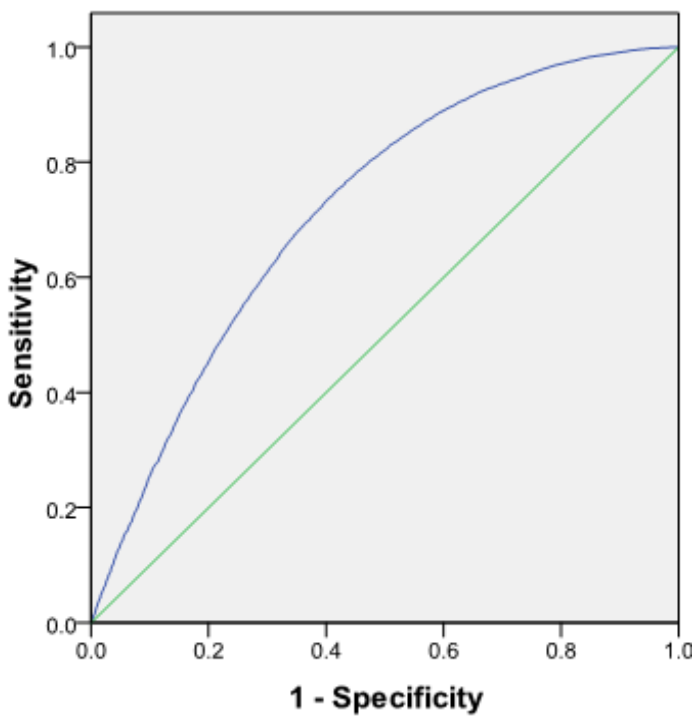

Logistic Model 3

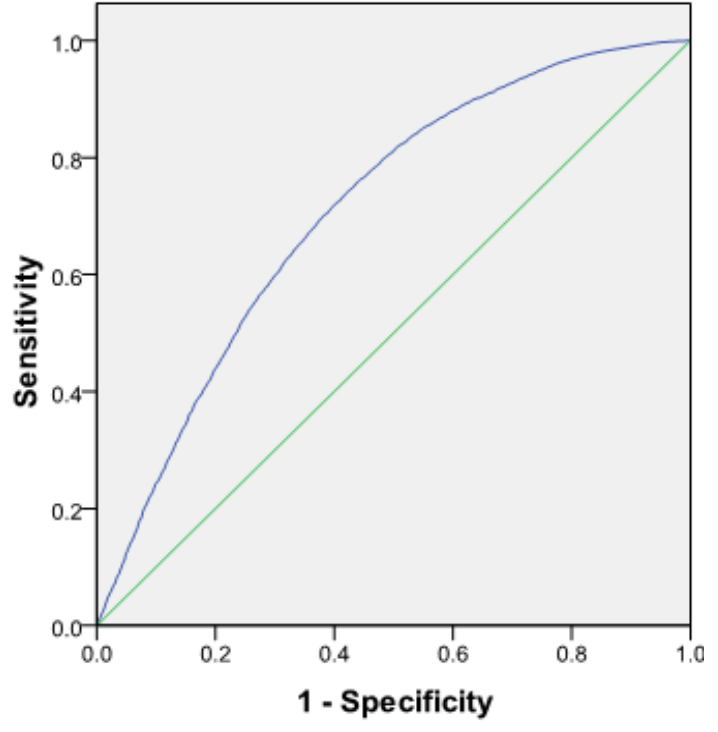

Logistic Model 2

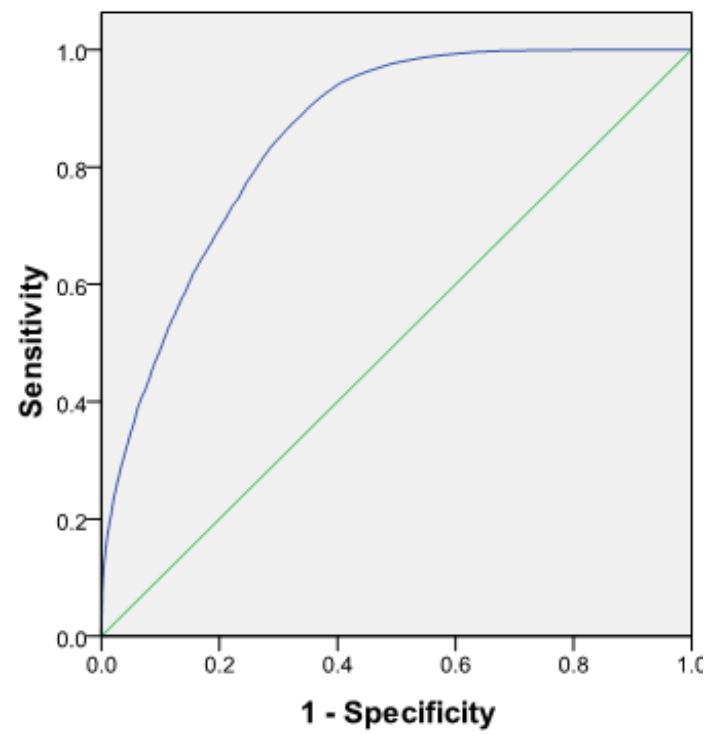

Logistic Model 4

Figure 1. ROC graphs of Logistic Regression models

\section{ANN Perceptron Classification and Back Propagation}

A perceptron is an arrangement of one McCulloch-Pitts neuron input layer feeding forward into one McCulloch-Pitts neuron output layer. A feedforward neural network of at least two layers is

characterized as a multilayer perceptron. Growing of the perceptron layers is utilized by splitting 
International Journal of Mathematical, Engineering and Management Sciences

Vol. 5, No. 6, 1170-1190, 2020

https://doi.org/10.33889/IJMEMS.2020.5.6.089

them into small linearly separable parts of data given to solve nonlinear separable problems (Widrow and Lehr, 1990). Hard-limiting function (step function) is the most preferred activation function for producing the outputs. The second most important function is a sigmoid function. Sigmoid function prevents the information of the inputs to overflow into the inner neurons. In modelling a complex data, step function is replaced with a sigmoid function. The combination of each individual perceptron with a sigmoid function combined with another series of perceptrons serves as the output of a multilayer perceptron. The architecture and design of the network is intended to be more flexible, for example, no immediate connection among input and target layers as well as associations between layers is assumed, the number of outputs need not be equivalent to the number of inputs, and there is no limitation on the number of hidden layers or units. Hidden units can even be more than or less than input and output units (Jabri and Flower, 1992).

The most widely used learning algorithm for training feedforward artificial neural networks is the backpropagation algorithm. In this technique, corresponding weights of previous layers neurons are multiplied separately along with receiving signals in the current layer. Inputs of one or more previous neurons are weighted independently and added. The weights between neurons are optimized using the backpropagation technique to generate the best network. Thus, multilayer perceptrons have two essential characteristics, generalization and fault tolerance. Neural networks are extremely tolerant to faults. This feature is also called graceful degradation (Mohr et al., 2000). Even if certain interconnections between certain neurons within the layers fail, the neural networks keep working. We developed ANN models in this research to fulfil the above characteristics.

\subsection{Survival Prediction using ANN Modelling}

When modelling non-linear data, artificial neural networks (ANN) are efficient estimators. Constructing an ANN requires minimum domain awareness in the fields of mathematics and statistics. The type of ANN used in this study is called the multilayer perceptron (MLP) or multilayer feed-forward network that propagates input signals forward and returns error signals. During the process the weights are changed to make prediction more accurate. This method is vulnerable to problematic overfitting. Training to the network is provided with some of the data values to prevent overfitting and then test its performance by testing the trained network with the values of the remaining data. We split the data into $70 \%-30 \% .70 \%$ for training our ANN models and the $30 \%$ data for testing and validation.

The ANN models consist of an input layer, a hidden layer and an output layer. Table 3 summarizes the training results of ANN models including specificity, sensitivity along with $95 \%$ confidence interval and overall accuracy of the model performance.

Table 3. ANN training results: sensitivity, specificity and accuracy

\begin{tabular}{|c|c|c|c|c|c|}
\hline \multirow{2}{*}{$\begin{array}{l}\text { Artificial Neural } \\
\text { Network } \\
\text { Models }\end{array}$} & \multicolumn{2}{|c|}{ Sensitivity } & \multicolumn{2}{|c|}{ Specificity } & \multirow[b]{2}{*}{ Accuracy } \\
\hline & Value & $\begin{array}{c}95 \% \\
\text { Confidence } \\
\text { Interval }\end{array}$ & Value & $\begin{array}{c}95 \% \\
\text { Confidence } \\
\text { Interval }\end{array}$ & \\
\hline Model 1 & $66.78 \%$ & $65.70-67.83$ & $70.76 \%$ & $70.19-71.31$ & $69.85 \%$ \\
\hline Model 2 & $67.25 \%$ & $66.19-68.27$ & $71.48 \%$ & $70.91-72.03$ & $70.46 \%$ \\
\hline Model 3 & $68.23 \%$ & $67.20-69.23$ & $72.08 \%$ & $71.51-72.63$ & $71.12 \%$ \\
\hline Model 4 & $88.95 \%$ & $88.27-89.59$ & $80.60 \%$ & $80.09-81.09$ & $82.80 \%$ \\
\hline
\end{tabular}


International Journal of Mathematical, Engineering and Management Sciences

Vol. 5, No. 6, 1170-1190, 2020

https://doi.org/10.33889/IJMEMS.2020.5.6.089

The specificity, sensitivity along with $95 \%$ confidence intervals and overall accuracy results of the developed ANN models when testing is summarized in Table 4.

Table 4. ANN testing results: sensitivity, specificity and accuracy

\begin{tabular}{|l|c|c|c|c|c|}
\hline \multirow{2}{*}{$\begin{array}{l}\text { Artificial Neural } \\
\text { Network } \\
\text { Models }\end{array}$} & Value & $\begin{array}{c}\text { S5\% Confidence } \\
\text { Interval }\end{array}$ & Value & $\begin{array}{c}\text { 95\% Confidence } \\
\text { Interval }\end{array}$ & \multirow{2}{*}{ Accuracy } \\
\cline { 2 - 5 } Model 1 & $66.18 \%$ & $64.52-67.80$ & $70.66 \%$ & $69.79-71.51$ & $69.63 \%$ \\
\hline Model 2 & $68.66 \%$ & $67.08-70.20$ & $72.00 \%$ & $71.14-72.84$ & $71.20 \%$ \\
\hline Model 3 & $66.87 \%$ & $65.26-68.43$ & $71.89 \%$ & $58.27-59.97$ & $70.67 \%$ \\
\hline Model 4 & $89.36 \%$ & $88.32-90.32$ & $80.97 \%$ & $80.20-81.72$ & $83.20 \%$ \\
\hline
\end{tabular}

Architecture of developed artificial neural network models with their ROC values along with specificity at $95 \%$ sensitivity are given in Table 5.

Table 5. Architecture of fitted ANN models and ROC AUC values

\begin{tabular}{|l|c|c|c|c|c|}
\hline \multirow{2}{*}{$\begin{array}{l}\text { Artificial Neural } \\
\text { Network }\end{array}$} & \multicolumn{3}{|c|}{ Architecture } & ROC & At 95\% sensitivity \\
\cline { 2 - 6 } Models & I & H & O & & Specificity \\
\hline Model 1 & 2 & 7 & 2 & $72.1 \%$ & $30 \%$ \\
\hline Model 2 & 6 & 3 & 2 & $73.1 \%$ & $32 \%$ \\
\hline Model 3 & 10 & 6 & 2 & $73.8 \%$ & $39 \%$ \\
\hline
\end{tabular}

$\mathrm{I}, \mathrm{H}, \mathrm{O}$ are the number of inputs, hidden and output nodes respectively.

From the training results we notice that the overall accuracy for model-3 to model-4 increased from $71.12 \%$ to $82.80 \%$. The neural network model-1 yielded a ROC area of $72.1 \%$ and a survival sensitivity of $95 \%$ yielded a specificity of $30 \%$, model-2 reported ROC area under curve of $73.1 \%$ and a survival sensitivity of $95 \%$ provided $32 \%$ specificity. The ROC region for the remaining two models is $73.8 \%$ and $87.4 \%$ respectively, and the $95 \%$ survival sensitivity yielded $39 \%$ and $66 \%$ specificity rates, respectively.

Comparing at $95 \%$ sensitivity, all four artificial neural network models has a higher specificity than logistic models. Figure 2 has the area under curve ROC graphs for the four neural network models. 


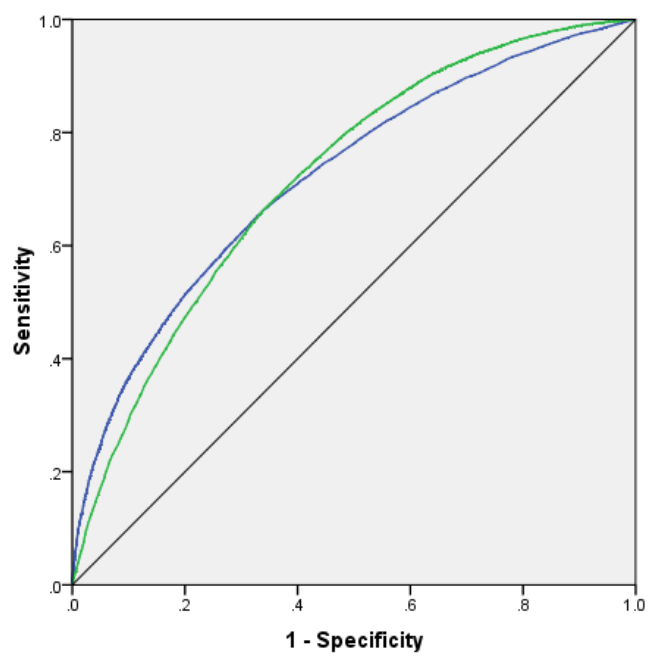

Neural Network Model-1

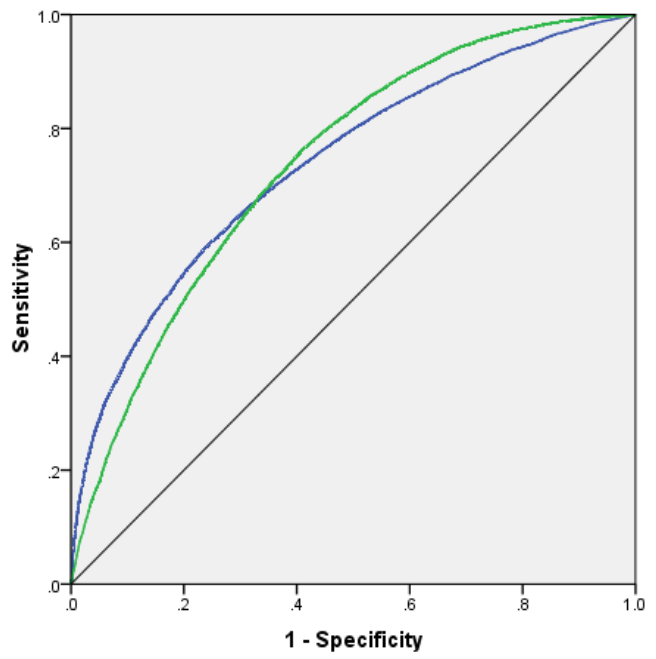

Neural Network Model-3

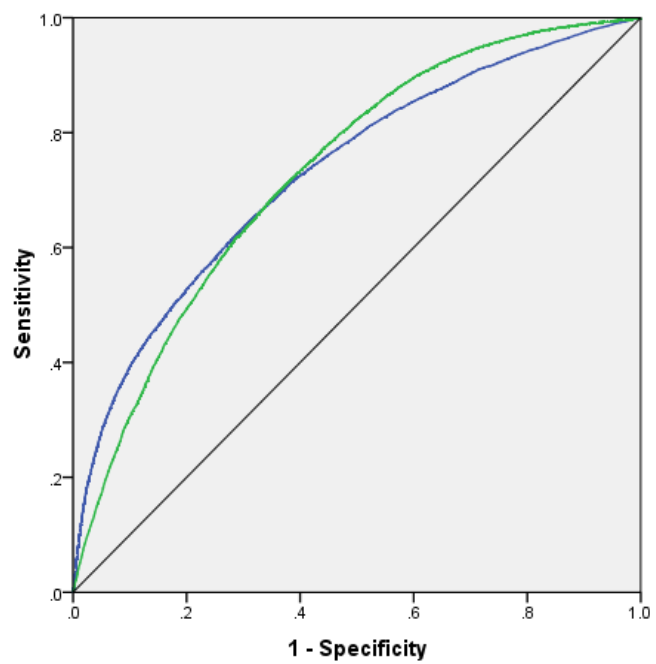

Neural Network Model-2

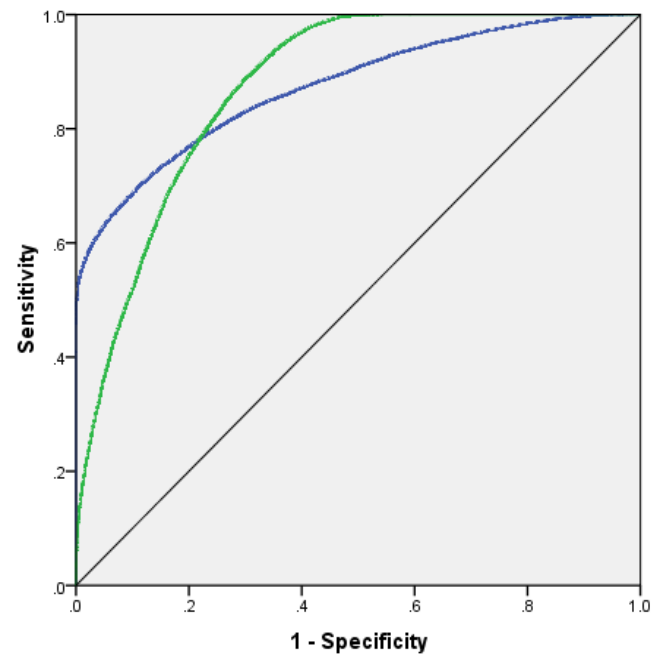

Neural Network Model-4

Figure 2. ROC graphs of ANN models

\section{Decision Tree Classification}

The effective classification approach is combining data mining tools with decision trees which assists physicians and medical specialists with a clear and easy understanding of classification rules. False positive and false negative decisions can be minimized with the help of methods of data mining. (Quinlan, 1987). 
International Journal of Mathematical, Engineering and Management Sciences

Vol. 5, No. 6, 1170-1190, 2020

https://doi.org/10.33889/IJMEMS.2020.5.6.089

A classification method or a classifier to assess acceptable action is referred as a Decision tree. Root, internal, or test nodes and leaf nodes form the core part of a simple decision tree. The final decisions for the target class are obtained on the leaf nodes by performing split tests within the internal nodes. The leaf node contains, in complex cases, target value or a probability vector for the result. (Zantema and Bodlaender, 2000).

The decision tree is usually composed of continuous or nominal attributes. One outcome is given for the target value when working with nominal attributes, while the continuous attributes will have two outcomes, one for each interval. Decision-makers typically prefer a less complex, decisionmaking tree. Any path from root to leaf of the decision tree generates a rule by calculating tests along the path that grant terminal node class prediction (Friedman et al., 1996).

\subsection{Splitting Techniques}

Decision trees also employ univariate separating, i.e., separating steps at each internal node is centred on the single attribute. Upon completion of splitting, the inducer is looking for the best attribute at the internal node. The splitting procedures are used in numerous ways, depending on the tree's initial measure and the level of the tree. For a univariate splitting, the choice of splitting criteria does not affect the performance of the tree.

Multivariate splits are usually based on the linear combination of the input variables. Methods used to find optimal splitting include the greedy search method (Breiman et al., 1984) linear programming (Duda et al., 2012), linear discriminant analysis (Friedman, 1977) and many more.

An alternative approach for maximizing the tree is to allow it to grow and replenish using certain methods of pruning. Pruning methods aim to yield simple trees at relatively small cost of reducing accuracy. There are different pruning methods, such as cost-complexion, reduced and minimal error, optimal, etc.

\subsection{Survival Prediction using Decision Trees}

The training and testing results of specificity, sensitivity along with $95 \%$ confidence intervals and overall accuracy results using both CHAID and CRT methods of the four decision tree models are summarized in Table 6 and Table 7.

Table 6. Decision trees training results: sensitivity, specificity and accuracy

\begin{tabular}{|l|l|c|c|c|c|c|}
\hline \multirow{2}{*}{$\begin{array}{l}\text { Decision Tree } \\
\text { Training }\end{array}$} & & \multicolumn{2}{|c|}{ Sensitivity } & \multicolumn{2}{|c|}{ Specificity } & \multirow{2}{*}{ Accuracy } \\
\cline { 2 - 6 } & & Value & $95 \%$ CI & Value & $95 \%$ CI & \\
\hline \multirow{2}{*}{$\begin{array}{l}\text { Decision Tree } \\
\text { Model 1 }\end{array}$} & CHAID & $64.77 \%$ & $63.73-65.8$ & $71.12 \%$ & $70.60-71.73$ & $69.6 \%$ \\
\cline { 2 - 7 } & CRT & $66.83 \%$ & $65.74-67.91$ & $70.75 \%$ & $70.19-71.30$ & $69.9 \%$ \\
\hline \multirow{2}{*}{$\begin{array}{l}\text { Mecision Tree } \\
\text { Model 2 }\end{array}$} & CHAID & $69.33 \%$ & $68.27-70.37$ & $71.21 \%$ & $70.65-71.76$ & $70.8 \%$ \\
\cline { 2 - 7 } $\begin{array}{l}\text { Decision Tree } \\
\text { Model 3 }\end{array}$ & CRT & $69.2 \%$ & $68.14-70.24$ & $71.2 \%$ & $70.63-71.74$ & $70.7 \%$ \\
\hline \multirow{2}{*}{$\begin{array}{l}\text { Decision Tree } \\
\text { Model 4 }\end{array}$} & CRAID & $67.59 \%$ & $66.58-68.59$ & $72.25 \%$ & $71.69-72.81$ & $71.1 \%$ \\
\cline { 2 - 7 } & CHAID & $66.22 \%$ & $65.22-67.21$ & $72.64 \%$ & $72.07-73.20$ & $70.9 \%$ \\
\cline { 2 - 7 } & CRT & $93.67 \%$ & $89.00-90.30$ & $80.14 \%$ & $79.63-80.63$ & $82.6 \%$ \\
\hline
\end{tabular}


International Journal of Mathematical, Engineering and Management Sciences

Vol. 5, No. 6, 1170-1190, 2020

https://doi.org/10.33889/IJMEMS.2020.5.6.089

Table 7. Decision trees testing results: sensitivity, specificity and accuracy

\begin{tabular}{|c|c|c|c|c|c|c|}
\hline \multirow[b]{2}{*}{$\begin{array}{l}\text { Decision Tree } \\
\text { Testing }\end{array}$} & & \multicolumn{2}{|c|}{ Sensitivity } & \multicolumn{2}{|c|}{ Specificity } & \multirow[t]{2}{*}{ Accuracy } \\
\hline & & Value & $\begin{array}{c}95 \% \\
\text { Confidence } \\
\text { Interval } \\
\end{array}$ & Value & $\begin{array}{c}95 \% \\
\text { Confidence } \\
\text { Interval } \\
\end{array}$ & \\
\hline \multirow{2}{*}{$\begin{array}{l}\text { Decision Tree } \\
\text { Model } 1\end{array}$} & CHAID & $63.66 \%$ & $62.07-65.22$ & $71.22 \%$ & $70.35-72.09$ & $69.3 \%$ \\
\hline & CRT & $67.33 \%$ & $65.66-68.97$ & $69.69 \%$ & $68.83-70.55$ & $69.2 \%$ \\
\hline \multirow{2}{*}{$\begin{array}{l}\text { Decision Tree } \\
\text { Model } 2\end{array}$} & CHAID & $68.63 \%$ & $66.97-70.24$ & $71.27 \%$ & $70.40-72.12$ & $70.7 \%$ \\
\hline & CRT & $67.24 \%$ & $65.56-68.87$ & $70.82 \%$ & $69.95-71.67$ & $70 \%$ \\
\hline \multirow{2}{*}{$\begin{array}{l}\text { Decision Tree } \\
\text { Model } 3\end{array}$} & CHAID & $66.32 \%$ & $64.75-67.85$ & $72.19 \%$ & $71.31-73.04$ & $70.7 \% \%$ \\
\hline & $\mathrm{CRT}$ & $65.11 \%$ & $63.55-66.65$ & $71.42 \%$ & $70.54-72.28$ & $69.8 \%$ \\
\hline \multirow{2}{*}{$\begin{array}{l}\text { Decision Tree } \\
\text { Model } 4\end{array}$} & CHAID & $88.2 \%$ & $87.10-89.21$ & $80.38 \%$ & $79.61-81.14$ & $82.4 \%$ \\
\hline & CRT & $94.02 \%$ & $93.19-94.76$ & $79.93 \%$ & $79.16-80.69$ & $83.2 \%$ \\
\hline
\end{tabular}

The results showed that in a CHAID decision tree, the overall accuracy for the model-3 increased from $71.10 \%$ to $82.6 \%$ for the model- 4 . Similarly, the accuracy jumps from $70.9 \%$ for model-3 to $83.2 \%$ for model-4 for a CRT based decision tree.

The $95 \%$ survival sensitivity has returned a specificity of 22, 25, 29 and 62 percent respectively for the four models. CHAID model-4 decision tree results have an overall accuracy of $82.6 \%$ with a specificity of $80.14 \%$ and a sensitivity of $89.67 \%$. These numbers suggest that model-4 of CHAID is a very well-performing model.

The ROC area under curve for the four models developed on CRT based tree are $71.9 \%, 72.8 \%$, $72.7 \%$ and $87.4 \%$ respectively. The $95 \%$ survival sensitivity has returned a specificity of 24,29 , 28 and 62 percent respectively for the four models. CRT model-4 decision tree tests have an overall accuracy of $82.2 \%$. The specificity and sensitivity for the model-4 are $79.86 \%$ and $93.62 \%$. These numbers indicate that model-4 CRT is a very well-performing model. ROC values of the developed decision tree models using CHAID and CRT are summarized the Table 8.

Table 8. ROC of Decision tree using CHAID and CRT

\begin{tabular}{|l|c|c|c|c|}
\hline & Model-1 & Model-2 & Model-3 & Model-4 \\
\hline CHAID & $72.0 \%$ & $73.2 \%$ & $73.6 \%$ & $87.6 \%$ \\
\hline CRT & $71.9 \%$ & $72.8 \%$ & $72.7 \%$ & $87.4 \%$ \\
\hline
\end{tabular}

Figure 3 has the ROC graphs for the decision trees developed using CHAID technique. From the results of Table 8, we notice that there is a significant increase in the area under curve for model-3 and model-4. Clearly, approximately there is an $88 \%$ chance that model-4 can distinguish between positive and negative cases.

Figure 4 has the ROC graphs for the decision trees developed using CRT technique. As observed in CHAID models, even in CRT based models, there is a significant increase in the area under curve for model-3 and model-4 with model-4 having an $87.4 \%$ chance of distinguishing between positive and negative cases. 

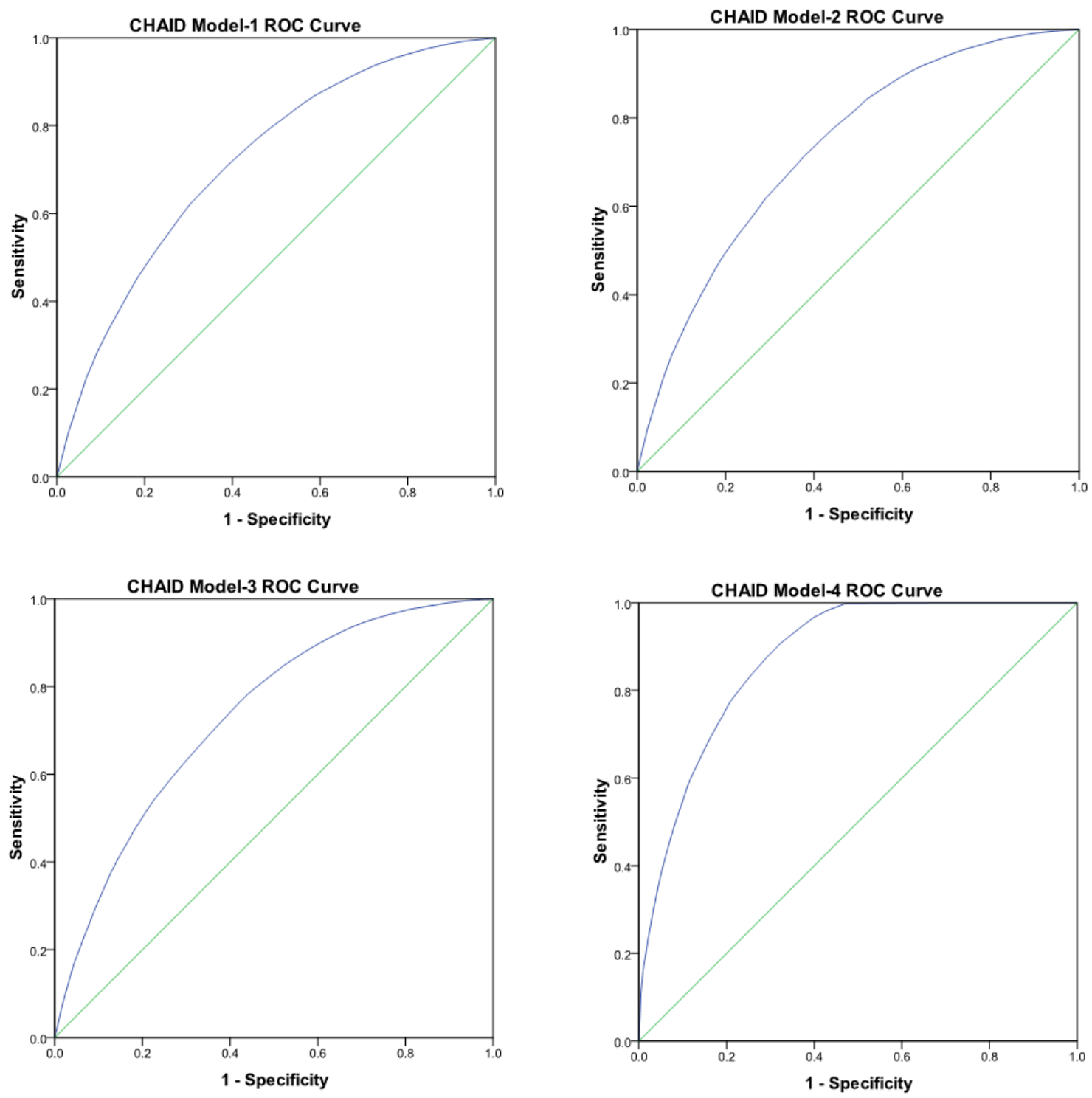

Figure 3. ROC areas for CHAID based Decision trees

By default, CHAID uses multiway splits while CRT uses binary splits. This disparity between the CRT and the CHAID affects even the decision tree structures. Segmentation or classification has various significant applications.

- When the dependent variable is categorical, CHAID, Chi-square Automatic Interaction Detector, splits the tree based on chi-square test. On the other hand, CRT uses impurity reduction as a measure to split.

- In order to produce a smaller tree rather than an exhaustive one, CHAID uses the forward stepwise stopping rule. CRT intentionally overfits and uses validation data to prune back to identify the best and smallest tree. 
International Journal of Mathematical, Engineering and Management Sciences

Vol. 5, No. 6, 1170-1190, 2020

https://doi.org/10.33889/IJMEMS.2020.5.6.089

Finally, if the aim is to identify or explain the relationship between a response variable and a collection of explanatory variables, one may prefer CHAID, while CRT is best suited for constructing a regression model. At this viewpoint, in this paper, we conclude that for the survival classification of breast cancer in women, CHAID decision tree performed marginally better than CRT.
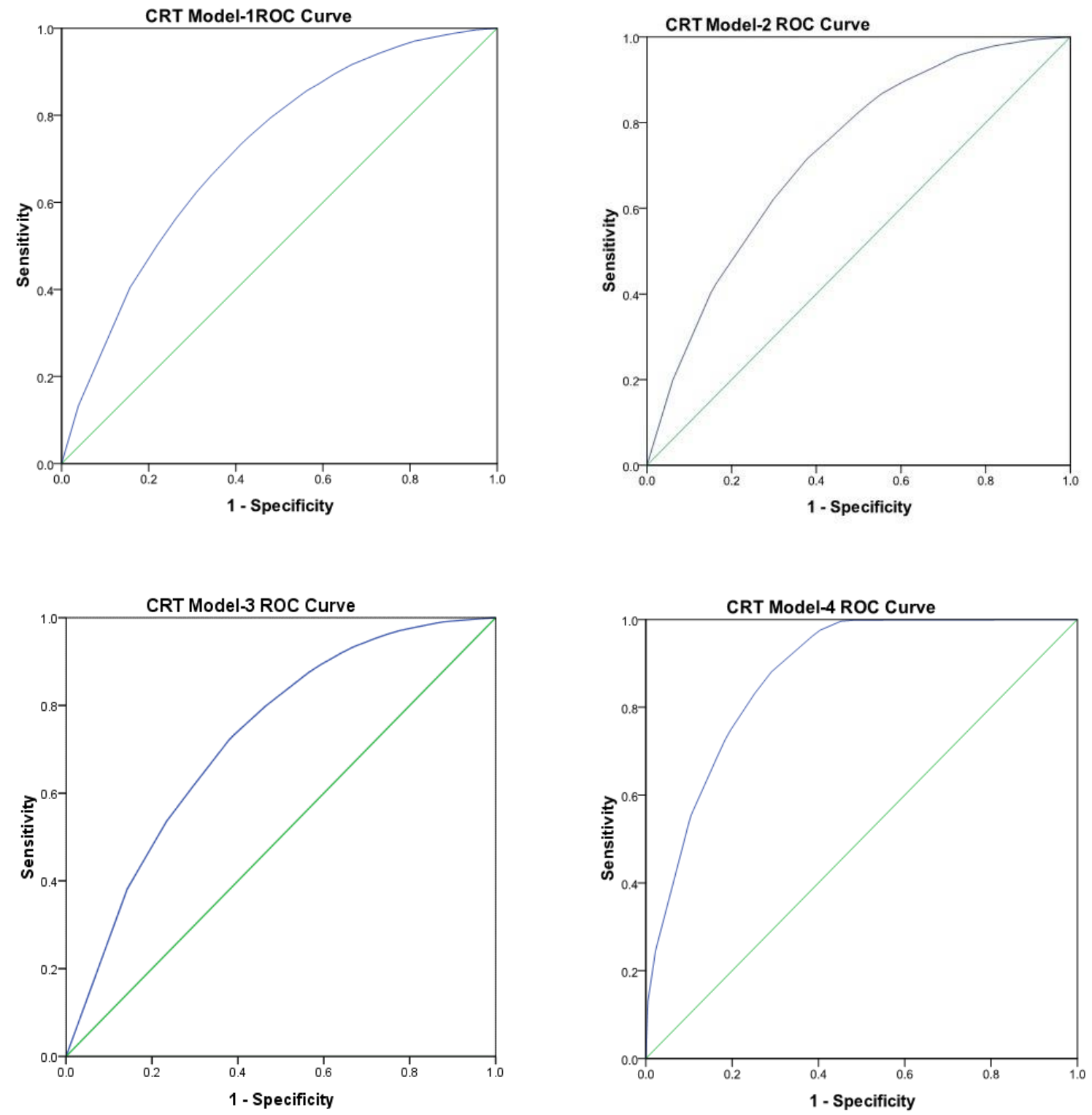

Figure 4. ROC areas for CRT based Decision trees 
International Journal of Mathematical, Engineering and Management Sciences

Vol. 5, No. 6, 1170-1190, 2020

https://doi.org/10.33889/IJMEMS.2020.5.6.089

\section{Performance Evaluation of Models}

One of four outcomes are possible when evaluating a predictive binary classification model:

(a) a true positive (TP) (b) a false positive (FP) (c) a true negative (TN) (d) a false negative (FN).

The central concern of implementing various modelling applications in this paper is to determine which of the techniques proposed would enhance predictive accuracy. Even a small fraction of a percent change will turn into substantial savings or increased revenues.

The performance of logistic regression models, artificial neural network models and decision tree models in this work is evaluated based on sensitivity, specificity, accuracy and ROC area under curve values. Sensitivity is the proportion of true positive elements that the model correctly classifies. Specificity is the proportion of true negatives that the model correctly classifies.

In general, we compare the area under the ROC curve, which is an easy way to test predictive binary classification models when the analyst or decision-maker does not have any knowledge about the cost or extent of classification errors. We compared the results of the four logistic models with the results of four artificial neural network models and four decision tree models, respectively.

Figure 5 and Figure 6 depicts the comparison of specificity and overall accuracy of the models developed. In comparing the specificity and overall accuracy of all models, model-4 clearly stands out as a better model. Models 1, 2 and 3 resulted with similar results of accuracy, specificity and ROC values.

Table 9 includes the performance assessment of logistic, artificial neural network, and decision tree techniques. Compared with logistic models, neural network and decision tree techniques reported almost the same overall accuracy for correct classification of breast cancer survival in women. Nonetheless, the specificity of the logistic model results is higher than the neural network and decision tree models. Models developed are ranked, given in Table 9, based on their performance and accuracy in classification. For all the four models, the area under the ROC curves of decision tree methods is comparatively higher than the other two techniques.

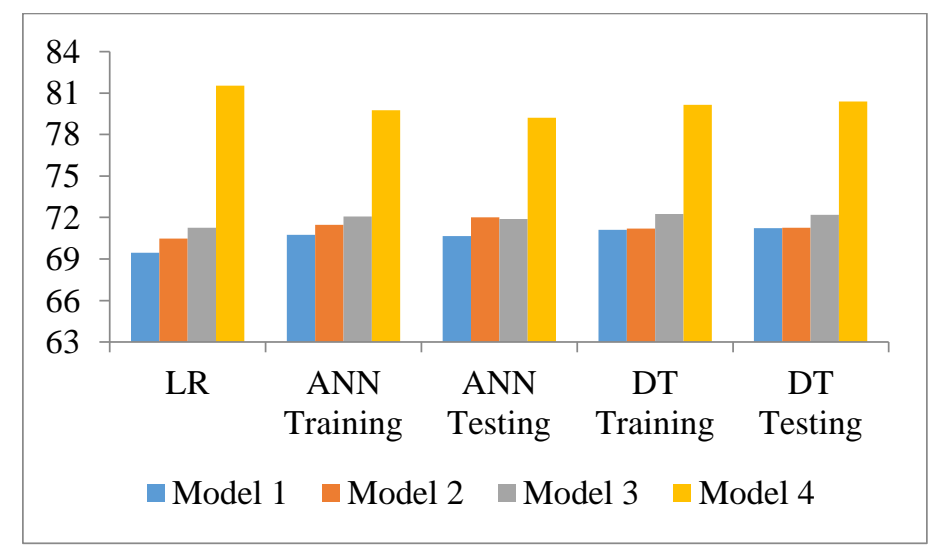

Figure 5. Specificity comparison of Logistic Regression, Artificial Neural Networks and Decision Tree models 
International Journal of Mathematical, Engineering and Management Sciences

Vol. 5, No. 6, 1170-1190, 2020

https://doi.org/10.33889/IJMEMS.2020.5.6.089

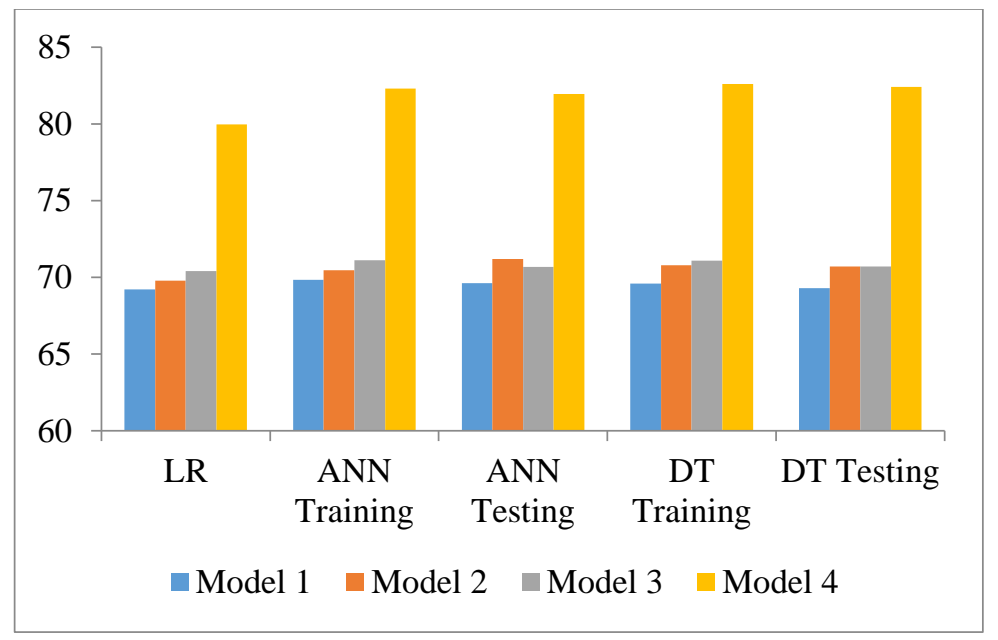

Figure 6. Comparison of overall accuracy of Logistic Regression, Artificial Neural Networks and Decision Tree models

Table 9. Performance evaluation of Logistic, ANN and decision tree models

\begin{tabular}{|c|c|c|c|c|c|c|c|c|c|c|}
\hline & \multicolumn{5}{|c|}{ Overall Accuracy } & \multicolumn{5}{|c|}{ Specificity } \\
\hline \multirow[t]{2}{*}{ Model } & LR & \multicolumn{2}{|c|}{ ANN } & \multicolumn{2}{|c|}{ CHAID } & LR & \multicolumn{2}{|c|}{ ANN } & \multicolumn{2}{|c|}{ CHAID } \\
\hline & & Train & Test & Train & Test & & Train & Test & Train & Test \\
\hline 1 & 69.2 & 69.85 & 69.63 & 69.6 & 69.3 & 69.45 & 70.76 & 70.66 & 71.17 & 71.22 \\
\hline 2 & 69.78 & 70.46 & 71.2 & 70.8 & 70.7 & 70.47 & 71.48 & 72 & 68.63 & 71.27 \\
\hline 3 & 70.42 & 71.12 & 70.67 & 71.1 & 70.7 & 71.26 & 72.08 & 71.89 & 72.25 & 72.19 \\
\hline 4 & 79.98 & 82.31 & 81.95 & 82.6 & 82.4 & 81.54 & 79.76 & 79.22 & 80.14 & 80.4 \\
\hline Rank & III & \multicolumn{2}{|c|}{ II } & \multicolumn{2}{|c|}{ I } & I & \multicolumn{2}{|c|}{ III } & \multicolumn{2}{|c|}{ II } \\
\hline
\end{tabular}

Table 10 provides the specifics of comparing ROCs of the three different approaches along with their ranking.

Table 10. ROC values of Logistic, ANN and Decision Tree models

\begin{tabular}{|l|c|c|c|}
\hline Models & LR & ANN & DT \\
\hline Model-1 & $68.8 \%$ & $72.1 \%$ & $72.0 \%$ \\
\hline Model-2 & $71.0 \%$ & $73.1 \%$ & $73.2 \%$ \\
\hline Model-3 & $71.8 \%$ & $73.8 \%$ & $73.6 \%$ \\
\hline Model-4 & $85.5 \%$ & $87.4 \%$ & $87.6 \%$ \\
\hline Rank & III & II & I \\
\hline
\end{tabular}

\section{Conclusion and Discussion}

This research aimed to compare breast cancer survival predictions of the artificial neural networks (ANN), decision trees, and logistic models. In the present research, we established models with the 
attributes of a woman with breast cancer, including age, tumor size and cancer stage, treatment administered, and duration. Four models with different attributable variables are developed and compared using logistic, ANN and decision trees (using CHAID and CRT algorithms). The degree of generalization (or the precision of predictive ability) was determined and the corresponding predictive abilities of the developed models are assigned a rank in this order. Model-4, Model-3, Model-2, Model1.

To maintain consistency in comparing these implemented models, we considered the accuracy of three techniques when classifying breast cancer survival results. The receiver operating characteristic (ROC) curves are plotted and estimated the area under the curves. The results indicated that Model- 4 yielded better performance using logistic regression, ANN, and decision tree methods. The model classification accuracy is obtained as $79.98 \%, 82.31 \%$ and $82.6 \%$ respectively for logistic regression, ANN and decision tree models. In the models developed using ANN and decision tree methods, we find no significant difference in the results obtained. However, the ANN method provided a better specificity when compared to the logistic and decision tree models at a 95\% sensitivity. In this study, the artificial neural networks and decision tree methods reported a modest improvement in the outcomes compared with logistic regression. Figure 7 is a comparison of the ROC curves of model-4 using all three techniques.

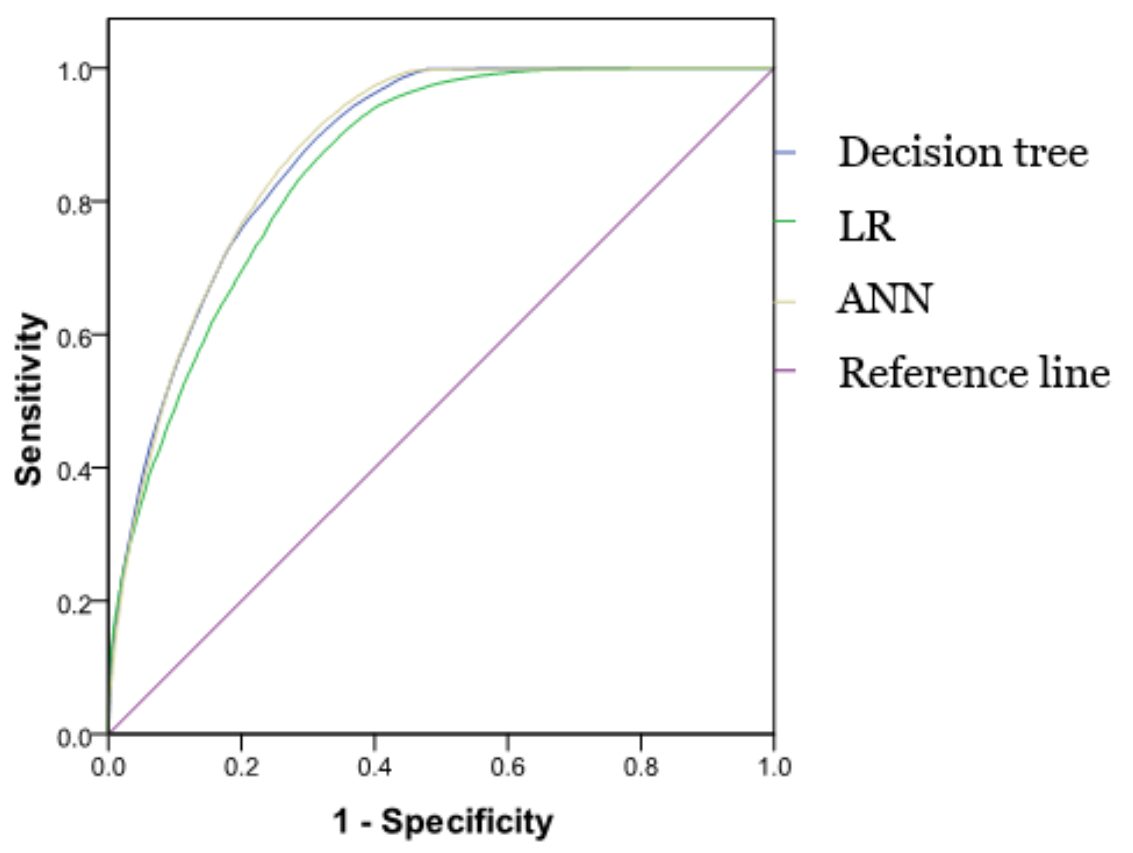

Figure 7. ROC comparison of the logistic regression, artificial neural network and decision tree models

We conclude in this analysis that ANN and decision tree models have a higher predictive probability compared to the logistic model. 
International Journal of Mathematical, Engineering and Management Sciences

Vol. 5, No. 6, 1170-1190, 2020

https://doi.org/10.33889/IJMEMS.2020.5.6.089

\section{Conflict of Interest}

The authors confirm that there is no potential conflict of interest to publish the paper in the journal.

\section{Acknowledgements}

This research paper is the revised and extended version of the chapter 6 of the first author Ph.D. dissertation. We would like to thank Dr. Gangaram Ladde for his valuable suggestions in completing this research work. We would also like to thank Ms. Saisumasri Komarraju, for her unconditional support in formatting this article for publication.

\section{References}

Agresti, A. (2010). Analysis of ordinal categorical data. John Wiley \& Sons. New Jersey.

Breiman, L., Friedman, J., Stone, C.J., \& Olshen, R.A. (1984). Classification and regression trees. Chapman $\&$ Hall/CRC press.

Duda, R.O., Hart, P.E., \& Stork, D.G. (2012). Pattern classification. John Wiley \& Sons. New York.

Friedman, J.H. (1977). A recursive partitioning decision rule for nonparametric classification. IEEE Transactions on Computers, 26(4), 404-408.

Friedman, J.H., Kohavi, R., \& Yun, Y. (1996, August). Lazy decision trees. In AAAI-96 Conference Proceedings (Vol. 1, pp. 717-724).

Gellar, J.E., Colantuoni, E., Needham, D.M., \& Crainiceanu, C.M. (2014). Variable-domain functional regression for modeling ICU data. Journal of the American Statistical Association, 109(508), 14251439.

Jabri, M., \& Flower, B. (1992). Weight perturbation: an optimal architecture and learning technique for analog VLSI feedforward and recurrent multilayer networks. IEEE Transactions on Neural Networks, 3(1), 154-157.

McFadden, D. (1973). Conditional logit analysis of qualitative choice behavior. In: Zarembka, P. (ed) Frontiers in Econometrics. Academic Press, New York, pp. 105-142.

Mohr, A.E., Riskin, E.A., \& Ladner, R.E. (2000). Unequal loss protection: graceful degradation of image quality over packet erasure channels through forward error correction. IEEE Journal on Selected Areas in Communications, 18(6), 819-828.

Mudunuru, V.R. (2016). Comparison of activation functions in multilayer neural networks for stage classification in breast cancer. Neural, Parallel, and Scientific Computations, 24, 83-96.

Quinlan, J.R. (1987). Simplifying decision trees. International Journal of Man-Machine Studies, 27(3), 221234.

Widrow, B., \& Lehr, M.A. (1990). 30 years of adaptive neural networks: perceptron, madaline, and backpropagation. Proceedings of the IEEE, 78(9), 1415-1442.

Zantema, H., \& Bodlaender, H.L. (2000). Finding small equivalent decision trees is hard. International Journal of Foundations of Computer Science, 11(02), 343-354. 


\section{Appendix}

\section{Data Source and Block Diagram}

- Breast Cancer Data has been requested from https://seer.cancer.gov/seertrack/data/request/

- Pre-process the data using excel (and macros) for missing values, removing the unwanted columns and prepare the columns identifying attributable variables, and by merging columns (if needed).

- Our Final dataset included the following variables: age, tumor size, stage of cancer, treatment, duration, and censor.

- Sample of our data is available upon request.

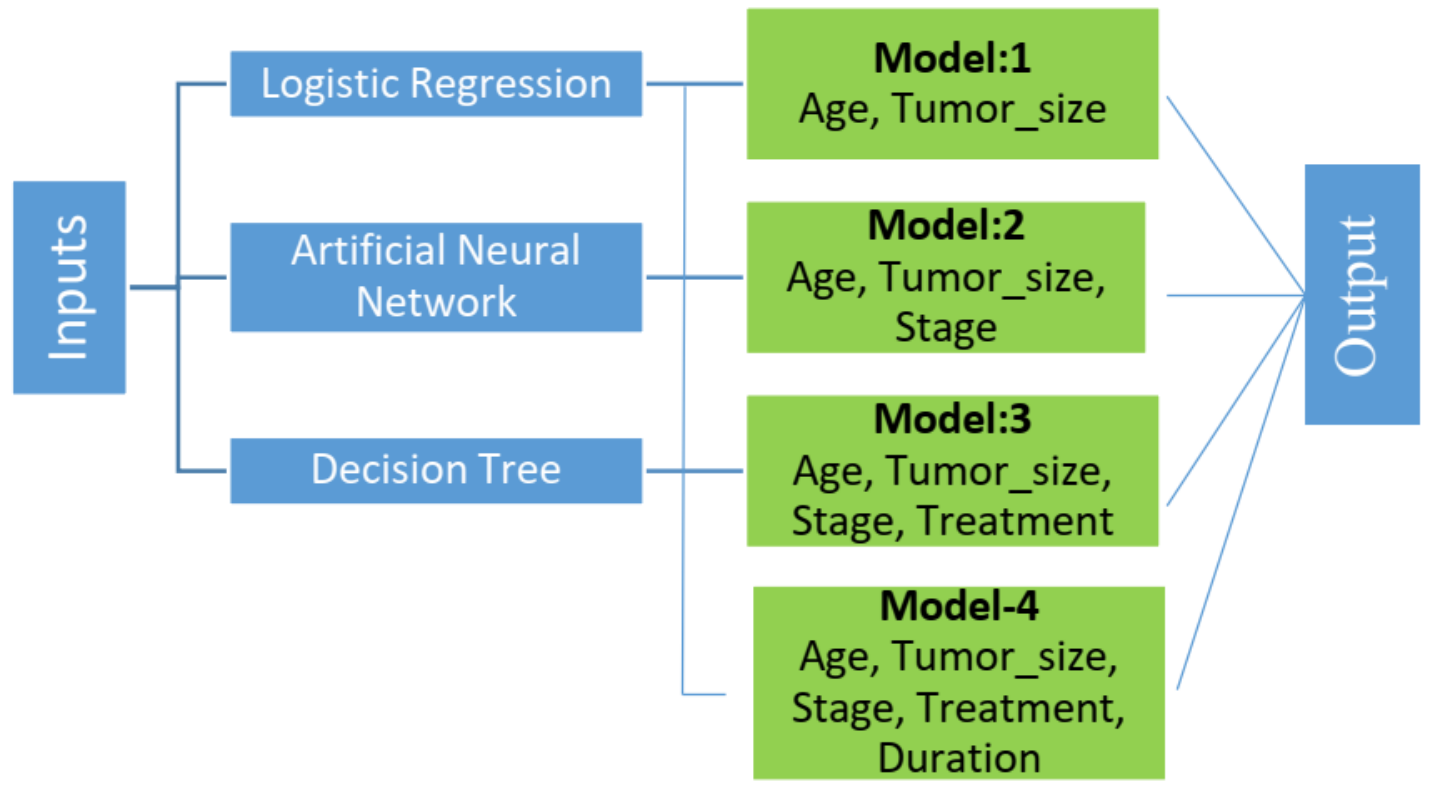

Figure 8. Block Diagram

Figure 8 is the complete block diagram of the research conducted in this paper.

\section{Software}

IBM SPSS Statistics, Version 22. 
International Journal of Mathematical, Engineering and Management Sciences

Vol. 5, No. 6, 1170-1190, 2020

https://doi.org/10.33889/IJMEMS.2020.5.6.089

\section{SPSS Set Up for Logistic Regression (LR) Models}

\section{Analysis $>$ Regression $>$ Binary Logistic}

Dependent: Censor

Covariates: Age, Tumor_size, Stage, Treatment, Duration

Method: Enter

Categorical

Under the list of Covariates, select Stage, Treatment as Categorical Covariates

\section{Save}

Predicted Valueg

$\square$ Probabilities

Influence

$\square \quad$ Cook's

Export model information to XML file

BROWSE and SAVE THE FILE ON YOUR COMPUTER.

$\square$ Include the covariance matrix

Options

Statistics and Plots

$\square \quad$ Clagsification plots

Display

(-) At each step

Probability for Stepwise

Entry: 0.05 Remoyal: 0.10

Classification cutoff: 0.5

Maximum Iterations: $\mathbf{2 0}$

$\bar{D}$ Include constant in model

Figure 9. SPSS set up for Logistic Regression Models

\section{ROC Curve for Logistic Regression}

\begin{tabular}{|c|}
\hline Analysis $>$ ROC Curve... \\
\hline $\begin{array}{l}\text { Test Variable: Predicted Probability } \\
\text { State Variable: CENSOR } \\
\text { Value of State Variable: } 1 \\
\text { Display } \\
\square \text { ROC Curve } \\
\square \text { With diagonal reference line } \\
\square \text { Standard error and confidence interval } \\
\text { Options... } \\
\text { Classification } \\
\odot \text { Include cutoff value for positive classification } \\
\text { Test Direction } \\
\odot \text { Larger test result indicate more positive test } \\
\text { Parameters for Standard Error of Area } \\
\text { Distribution Assumption: Nonparametric } \\
\text { Confidence Level: } 95 \% \\
\text { Missing Values } \\
\odot \text { Exclude both user-missing values and system missing values }\end{array}$ \\
\hline
\end{tabular}

Figure 10. ROC Curve for Logistic Regression 
International Journal of Mathematical, Engineering and Management Sciences

Vol. 5, No. 6, 1170-1190, 2020

https://doi.org/10.33889/IJMEMS.2020.5.6.089

\section{SPSS Set Up for Decision Tree (DT) Models}

\section{Analysis \\ Classify \\ Tree}

Dependent Variable: CENSOR

Independent Variables: Age, Tumor_size, Duration, Stage, Treatment

Growing Method

CHAID and CRT

\begin{tabular}{|l|}
\hline Output... \\
\hline Tree - Tree: \\
Display \\
Orientation: \\
$\odot$ Top down \\
Node Contents: \\
$\odot$ Table \\
Scale: \\
$\odot$ Automatic \\
$\square$ Independent variable statistics \\
$\square$ Node definitions \\
Statistics - Model: \\
$\square$ Summary \\
$\square$ Risk \\
$\square$ Classification Table \\
\hline
\end{tabular}

\section{Validation...}

$\odot$ Split-sample validation

Case Allocation

() Use random assignment

Training Sample (\%): 70

Display Results For

() Training and test samples

\section{Criteria.}

\section{CHAID}

Growth Limits

Maximum Tree Depth

(- A Automatic

Minimum Number of Cases

Parent Node: 100

Child Node: 50

CHAID

Significance Level For

Splitting Nodes: 0.05

Merging Categories: 0.05

Model Estimation

Maximum Number of Iterations: 100

Minimum Change in Expected Cell Frequencies: 0.001

Chi-square Statistic

(-) Pearson

$\square$ Adjust significance values using

Intervals Bonferroni method

Intervals for Scale Independent Variables

( ) Fixed Number Value: 10

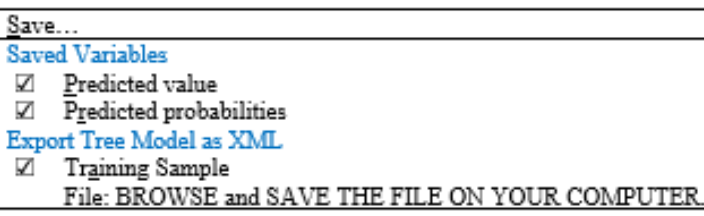

Figure 12. SPSS set up for Decision Tree Models 
International Journal of Mathematical, Engineering and Management Sciences

Vol. 5, No. 6, 1170-1190, 2020

https://doi.org/10.33889/IJMEMS.2020.5.6.089

\section{ROC Curve for Decision Tree}

\section{Analysis $>$ ROC Curve...}

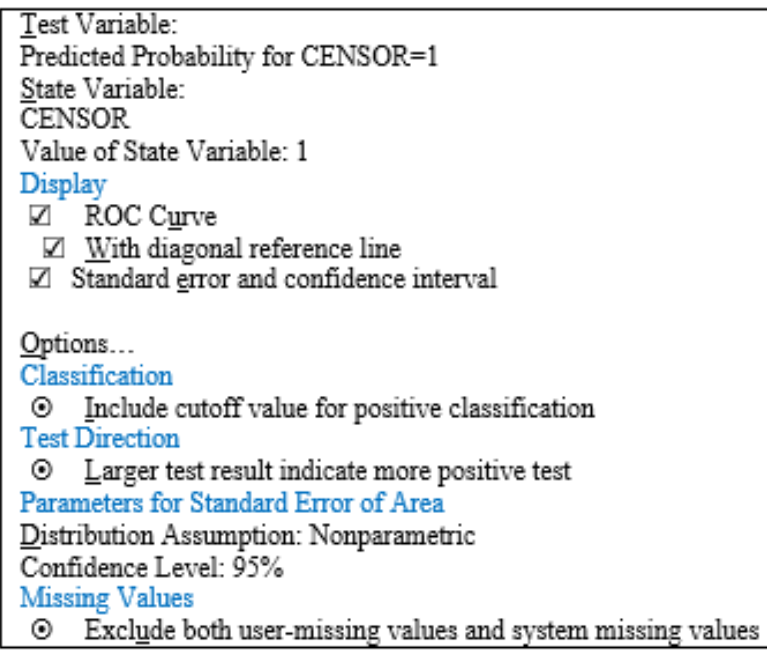

Figure 13. ROC curve for Decision Tree

Figure 9 explains the steps involved in modelling a logistic regression. Figure 10 gives the steps for generating ROC curves for a logistic regression model.

Figure 11 is a step-by-step break down for performing a neural network analysis along with generating ROC curves.

Figure 12 has the steps involved in performing a decision tree analysis by both CHAID and CRT techniques. Figure 13 gives the steps for generating ROC curves for a decision tree model.

\section{Models Comparison}

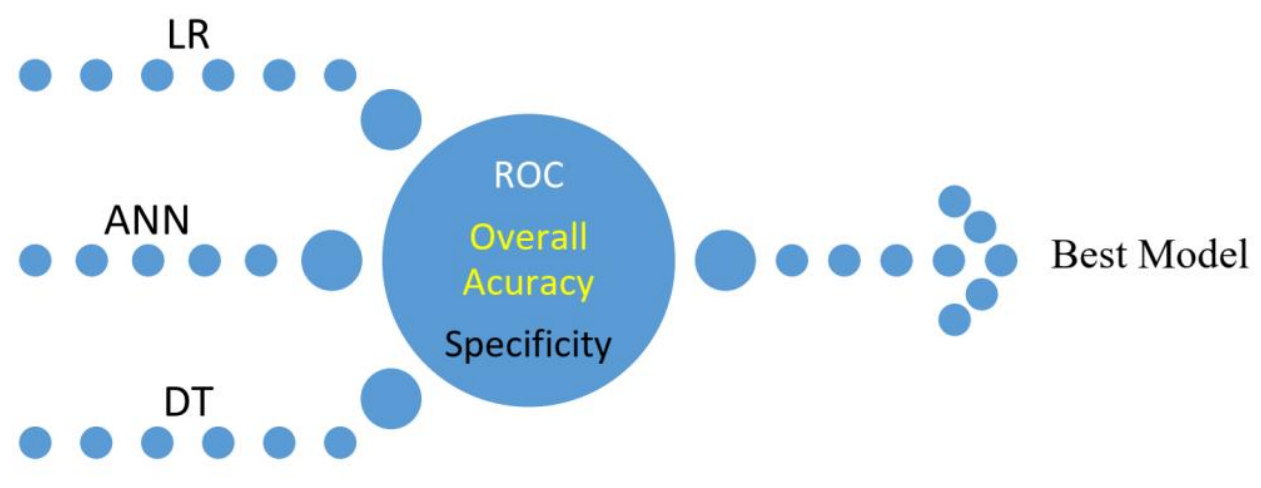

Figure 14. Models Comparison 
International Journal of Mathematical, Engineering and Management Sciences

Vol. 5, No. 6, 1170-1190, 2020

https://doi.org/10.33889/IJMEMS.2020.5.6.089

Figure 14 above is the comparison of the models with evaluating ROC, overall accuracy and specificity factors in order to identify the best classification model. 